\title{
The vignette for the V13N6 issue
}

\section{Fractionation and identification of 9c, 11t, 13t-conjugated linolenic acid as an activator of PPAR $\alpha$ in bitter gourd (Momordica charantia L.)}

Bitter gourd (Momodica charantia) contains an active principle which activates peroxisome proliferator receptor (PPARs) $\alpha$ and $\gamma[1]$. To identify the active compound, the extracts of bitter gourd was fractionated by silica gel column and then by preparative HPLC. The transactivation assay by using a clone of CHOK 1 cells with an alkaline phosphatase receptor and a chimeric receptor, GAL4-rPPAR $\alpha$ LBD was used to track the active component. By Mass, NMR and IR spectroscopy, 9cis, 11 trans, 13trans-conjugated linolenic acid (9c, $11 \mathrm{t}, 13 \mathrm{t}-\mathrm{CLN}$ ) was identified as a PPAR $\alpha$ activator. The bitter gourd contains about $7.1 \mathrm{~g} / \mathrm{kg}$ of $9 \mathrm{c}, 11 \mathrm{t}, 13 \mathrm{t}-\mathrm{CLN}$, which could play a key role in the control of lipid and glucose homeostasis and inflammatory response [2].

\section{Kinase activity and subcellular distribution of a chimeric green fluorescent protein-tagged Janus kinase 2}

Janus kinase 2 (JAK2) is an important intracellular signal transducer for many cytokines and hormones. Here, Lee et al. generated expression vectors encoding chimeric protein containing an enhanced green fluorescent protein (EGFP) linked to rat JAK2 (EGFP/rJAK2). They characterized the function and subcellular localization of chimeric EGFP/rJAK2 protein in transfected cells. They showed that alteration of one amino acid in the rJAK2(K882E) led to the change of subcellular localization of rat JAK2 in transfected cells [3]. Their studies provide insight into the controversy surrounding the subcellular localization of JAKs.

\section{Platelet microparticle in leukocyte and neutrophil activation}

During the inflammation process, the interaction of neutrophils with activated platelets is coordinated by an adhesion cascade [4]. Adhesion between platelets and leukocytes may represent an important process in hemostasis and thrombosis. Platelet activation is known to result in the release of microparticles by shedding of membranes from the surface [4]. Recently Simon et al. reported that platelet GPIb $\alpha$ is the counterreceptor for Mac-1 on leukocyte, accounting for firm adhesion and trans-platelet migration of leukocytes on vascular thrombus [5]. However, the role of GPIb $\alpha$ in the interplay between platelet MP and leukocyte has not been addressed. Results of this study demonstrated that the level of their aggregation can be reduced by GPIb blocking antibodies, AP1 and SZ2, but not by anti-CD18 mAb. The GPIb blocking antibodies also decreased platelet MP-mediated neutrophil activation, including $\beta 2$ integrin expression, adherence-dependent superoxide release and platelet MP-mediated neutrophil adherence to immobilized fibrinogen. This data provide evidence for the involvement of GPIbMac-1 interaction in the cross-talk between platelet MPs and neutrophils [6].

\section{Methamphetamine-induced behavioral sensitization in mice}

Endogenous opioids have been suggested to play a key role in mediating the rewarding effects of psychostimulants such as methamphetamine (METH). Although agonists of both $\delta$ - and $\mu$ opioid receptors exhibit rewarding property, only $\mu$ - but not $\delta$-opioid receptors play a major role in modulating mesolimbic dopamine system [7]. Furthermore, the effects of $\mu$-opioid receptor are greatly enhanced after repeated AMPH or METH treatment as indicated in the increase in dopamine releasing [8], suggesting functional alterations in $\mu$ opioid receptors. In this issue, Chiu et al. presented evidence supporting the notion that alterations in $\mu$-opioid receptor are associated with the induction and expression of METH-induced behavioral sensitization [9]. 
Induction of apoptosis and inhibition of cell adhesive and invasive effects by transhinone IIA in acute promyelocytic leukemia cells in vitro

Transhinone IIA, a diterpene quinine isolated from Chinese herb, has been used for the treatment of inflammatory diseases. Transhinone IIA caused anti-proliferation and apoptosis activities [10]. The apoptotic activity was shown by the activation of caspase-3, down-regulaton of antiapoptotic protein bcl-2 and bcl-xl, up-regulation of proapoptotic protein bax and disruption of mitochondria membrane potential. Furthermore, treatment of leukemia NB4 cells with transhinone IIA, decreased cell adhesion and invasion. It suggests that transhinone IIA could be a potential anti-leukemia drug [11].

\section{Cyclooxygenase 2 expression in cervical cancer}

Angiogenesis is essential for growth and development of solid tumors [12]. Recently, it has been reported that cyclooxygenase 2 (COX-2) works on angiogenesis associated with tumor growth and advancement including ovarian serous carcinomas [13]. Fujimoto et al. [14] therefore studied the clinical implications of COX-2 expression related to angiogenesis in uterine cerrical cancers. $\mathrm{COX}-2$ levels increase with cancer advancement, and the prognosis of the 30 patients with high COX-2 expression in uterine cervical cancers was poor $(60 \%)$, while the 24 -month survival rate of the other 30 patients with low COX-2 expression was $90 \%$. Furthermore, COX-2 levels significantly correlated with VEGF levels in uterine cervical cancers. VEGF associated with COX-2 might work on angiogenesis in cancer advancement.

\section{LK-4, an analog of dextromethorphan attenuates LPS-induced sepsis}

Dextromethorphan (DM), a dextrorotatory morphinan, is wildly used clinically as a non-opioid antitussive, and the neuroprotective effect elicited by femtomolar concentrations of DM is mediated through the inhibition of lipopolysaccharide (LPS)induced pro-inflammatory factors, especially superoxide [15]. LK-4, an analog of DM, has greater potency of anticonvulsant/neuro-protective actions than DM has [16]. Jiau et al. [17] found that treatment with LK-4 significantly attenuates the deleterious hemodynamic changes in rats treated with LPS. The induction of inducible NO synthase and the overproduction of $\mathrm{NO}$ and superoxide anions by LPS are also reduced by LK-4. These results suggest that the beneficial effects of LK-4 on LPS-induced sepsis results from its anti-inflammatory and antioxidant effects.

\section{The mortality of MOP3-deficient mice with a systemic functional failure}

Mop3, known as brain and muscle Arnt-like protein-1 (BMAL1), has been shown as an essential component of the master circadian pacemaker in mammals. Whether Mop3 or circadian rhythm also participates in regulation of other physiological function of mice such as life span is largely unknown. Recently, Ripperger and Schibler found that MOP3 directly bound to multiple E-box motifs of the liver-enriched transcriptional activator protein DBP and regulated Dbp expression in a circadian manner [18]. In this issue, Sun et al. [19] reported that MOP3-deficient mice had significantly reduced body weight and showed remarkable mortality around 6 months of age. Using the same animal model, they found that MOP3-deficient mice had significantly reduced levels of B cells in the peripheral blood, spleen and bone marrow [20]. These results suggest that either Mop3 has additional regulatory role other than circadian control or circadian rhythm itself may have subtle effect on a variety of physiological processes.

\section{Association of a BTLA gene polymorphism with the risk of rheumatoid arthritis}

Rheumatoid arthritis (RA) is an autoimmune disease characterized by joint destruction. Its pathogenesis is not yet clear, but the involvement of Th1 cells have been raised [21]. $B$ and $T$ lymphocyte attenuator (BTLA) is the third inhibitory receptor on $\mathrm{T}$ cells reported, which is believed to be involved in suppressing lymphocyte activation [22]. The involvement of BTLA in RA has been suspected due to the importance of $\mathrm{T}$ cell regulation in autoimmue disease. The potential 
association between BTLA gene and the development of RA has now been tested using a genetic case-control association study that compares a single nucleotide polymorphism (SNP) in the exon 5 of BTLA gene in RA patients and normal individuals in Taiwan [23]. The results show a statistically significant difference between the RA and the control groups, thus revealing a link between BTLA and RA.

\section{Hepatitis C virus NS4A inhibits cap-dependent and the viral IRES-mediated translation through interacting with eukaryotic elongation factor $1 \mathrm{~A}$}

Human hepatitis $\mathrm{C}$ virus (HCV) is the major causative agent of human chronic hepatitis and closely associated with hepatocellular carcinoma. Nonstructural protein 4A (NS4A) of $\mathrm{HCV}$ has been shown to inhibit protein synthesis. However, NS4A does not affect the steady state level of canonical translational factors including eIF4G, eIF4E, PABP, and 4E-BP1 [24]. Therefore, the mechanism of NS4A involved in the translational inhibition remains unclear. Kou et al. [25] report that NS4A specifically interacts with host eEF1A protein by GST pull down assay and LC-MS/MS analysis. They further demonstrate that the inhibitory effect of NS4A on both host cap-dependent and viral IRES-mediated translation is via its interaction with eEF1A in both culture system and in-vitro-translation system. Whether this inhibitory effect of NS4A contributes to the pathogenesis of $\mathrm{HCV}$ infection or escape of immune responses, leading to chronic infection requires further study.

\section{References}

1. Chao C.Y. and Hung C.J., Bitter gourd (Momodica charantia) extract activates peroxisome proliferators-activated receptors and upregulates the expression of the acyl CoA oxidase gene in H4IIEC3 hepatoma cells. J. Biomed. Sci. 10: 782-789, 2003.

2. Chuang C.Y., Hsu C., Chao C.Y., Wein Y.S., Kuo Y.H. and Huang C.J., Fractionation and identification of 9c, 11t, 13t-conjugated linolenic acid as an activator of PPAR $\alpha$ in bitter gourd (Momordica charantia L.). J. Biomed. Sci. (this issue doi 10.1007/s11373-006-9109-3).

3. Lee S. and Duhe R.J., Kinase activity and subcellular distribution of a chimeric green fluorescent protein-tagged Janus Kinase 2. J. Biomed. Sci. (this issue doi 10.1007/ s11373-006-9111-9).
4. Barry O.P. and FitzGerald G.A., Mechanisms of cellular activation by platelet microparticles. Thromb. Haemost. 82: 794-800, 1999.

5. Simon D.I., Chen Z., Xu H., Li C.Q., Dong J., McIntire L.V., Ballantyne C.M., Zhang L., Furman M.I., Berndt M.C. and Lopez J.A., Platelet glycoprotein ibalpha is a counterreceptor for the leukocyte integrin Mac-1 (CD11b/ CD18). J. Exp. Med. 192: 193-204, 2000.

6. Lo S.C., Hung C.Y., Lin D.T., Peng H.C. and Huang T.F., Involvement of platelet glycoprotein $\mathrm{Ib}$ in platelet microparticle mediated neutrophil activation. J. Biomed. Sci. (this issue doi 10.1007/s11373-006-9107-5).

7. Schad C.A., Justice J.B. and Holtzman S.G., Differential effects of delta- and mu-opioid receptor antagonists on the amphetamine-induced increase in extracellular dopamine in striatum and nucleus accumbens. J. Neurochem. 67: 22922299, 1996.

8. Yokoo H., Yamada S., Yoshida M., Tanaka T., Mizoguchi K., Emoto H., Koga C., Ishii H., Ishikawa M. and Kurasaki N., Effect of opioid peptides on dopamine release from nucleus accumbens after repeated treatment with methamphetamine. Eur. J. Pharmacol. 256: 335-338, 1994.

9. Chiu C.T., Ma T. and Ho I.K., Methamphetamine-induced behavioral sensitization in mice: alterations in u-opioid receptor. J. Biomed. Sci. (this issue doi 10.1007/s11373-0069102-x).

10. Yang L.J., Jeng C.J., Kung H.N., Chang C.C., Wang A.G., Chau G.Y., Don M.J. and Chau Y.P., Tanshinone IIA isolated from Salvia miltiorrhiza elcits the cell death of human endothelial cells. J. Biomed. Sci. 12: 347-361, 2005.

11. Liu J.J., Lin D.J., Liu P.O., Huang M., Li X.D. and Huang R.W., Induction of apoptosis and inhibition of cell adhesive and invasive effects by tanshinone IIA in acute promyelocytic leukemia cells in vitro. J. Biomed. Sci. (this issue doi 10.1007/s11373-006-9110-x).

12. Folkman J., Tumor angiogenesis. Adv. Cancer Res. 43: 175-203, 1985.

13. Ali-Fehmi R., Che M., Khalifeh I., Malone J.M., Morris R., Lawrence W.D. and Munkarah A.R., The effect of cyclooxygenase-2 expression on tumor vascularity in advanced stage ovarian serous carcinoma. Cancer 98: 1423-1429, 2003.

14. Fujimoto J., Toyoki H., Sato E., Sakaguchi H., Jahan I., Alam S.M. and Tamaya T., Expression of cyclooxygenase2 related to angiogenesis in uterine cervical cancers. J. Biomed. Sci. (this issue doi 10.1007/s11373-006-9114-6).

15. Li G., Cui G., Tzeng N.S., Wei S.J., Wang T., Block M.J. and Hong J.S., Femtomolar concentrations of dextromethorphan protect mesencephlic dopaminergic neurons from inflammatory damage. FASEB J. 19: 489496, 2005.

16. Kim H.C., Nabeshima T., Jhoo W.K., Ko K.H., Kim W.K., Shin E.J., Cho M. and Lee P.H., Anticonvulsant effects of new morphinan derivatives. Bioorg. Med. Chem. Lett. 11: 1651-1654, 2001.

17. Jiau S.S., Cheng P.Y., Lee Y.M., Huang W.H., Ko Y.F. and Yen M.H., Benefical effects of LK-4, an analog of dextromethorphan on lipopolysaccharide-induced sepsis in rats. J. Biomed. Sci. (this issue doi 10.1007/s11373-0069115-5).

18. Ripperger J.A. and Schibler U., Rhythmic CLOCKBMAL1 binding to multiple E-box motifs drives circadian Dbp transcription and chromatin transitions. Nat. Genet. 38: 369-374, 2006. 
19. Sun Y., Yang Z., Niu Z., Wang W., Peng J., Li Q., Ma M.Y. and Zhao Y., The mortality of MOP3 deficient mice with a systemic functional failure. J. Biomed. Sci. (this issue doi 10.1007/s11373-006-9108-4).

20. Sun Y., Yang Z., Niu Z., Peng J., Li Q., Xiong W., Langnas A.N., Ma M.Y. and Zhao Y., MOP3, a component of the molecular clock, regulates the development of B cells. Immunology 2006 Aug 22. [Epub ahead of print].

21. Simon A.K., Seipelt E. and Sieper J., Divergent T-cell cytokine patterns in inflammatory arthritis. Proc. Natl. Acad. Sci. USA 91: 8562-8566, 1994.

22. Watanabe N., Gavrieli M., Sedy J.R., Yang J., Fallarino F., Loftin S.K., Hurchla M.A., Zimmerman N., Sim J., Zang X., Murphy T.L., Russell J.H., Allison J.P. and Murphy K.M., BTLA is a lymphocyte inhibitory receptor with similarities to CTLA-4 and PD-1. Nat. Immunol. 4: 670-679, 2003

23. Lin S.C., Kuo C.C. and Chan C.H., Association of a BTLA gene polymorphism with the risk of rheumatoid arthritis. J. Biomed. Sci. (this issue doi 10.1007/s11373-006-9113-7).

24. Kato J., Kato N., Yoshida H., Ono-Nita S.K., Shiratori Y. and Omata M., Hepatitis C virus NS4A and NS4B proteins suppress translation in vivo. J. Med. Virol. 66: 187-199, 2002.

25. Kou Y.H., Chou S.M., Wang Y.M., Chang Y.T., Huang S.Y., Jung M.Y., Huang Y.H., Chen M.R., Chang M.F. and Chang S.C., Hepatitis C virus NS4A inhibits capdependent and the viral IRES-mediated translation through interacting with eukaryotic elongation factor $1 \mathrm{~A}$. J. Biomed. Sci. (this issue doi 10.1007/s11373-006-9104-8). 